\title{
Extended Pneumocephalus after Drainage of Chronic Subdural Hematoma Associated with Intracranial Hypotension : Case Report with Pathophysiologic Consideration
}

\author{
Hee Sup Shin, M.D., Ph.D., Seung Hwan Lee, M.D., Ph.D., Hak Cheol Ko, M.D., Jun Seok Koh, M.D., Ph.D. \\ Deapartment of Neurosurgery, Kyung Hee University Hospital at Gangdong, College of Medicine, Kyung Hee University, Seoul, Korea
}

Chronic subdural hematoma (SDH) is a well-known disease entity and is traditionally managed with surgery. However, when associated with spontaneous intracranial hypotension $(\mathrm{SIH})$, the treatment strategy ought to be modified, as classical treatment could lead to unwanted consequences. A 59-year-old man presented with a case of SIH that manifested as a bilateral chronic SDH. He developed fatal extensive pneumocephalus and SDH re-accumulation as a complication of burr-hole drainage. Despite application of an epidural blood patch, the spinal cerebrospinal fluid leak continued, which required open spinal surgery. Chronic SDH management should not be overlooked, especially if the exact cause has not been determined. When chronic SDH assumed to be associated with $\mathrm{SIH}$, the neurosurgeon should determine the exact cause of SIH in order to effectively correct the cause.

Key Words : Intracranial hypotension · Chronic subdural hematoma · Pneumocephalus.

\section{INTRODUCTION}

Spontaneous intracranial hypotension $(\mathrm{SIH})$ is a rare condition with a reported incidence of about 5 per 100000 individuals ${ }^{4)}$. It is typically characterized by low cerebrospinal fluid (CSF) pressure, diffuse pachymeningeal enhancement, and postural orthostatic headache which gradually increases and can occur without a history of trauma or a dural manipulation procedure. SIH is caused by leakage of CSF from a tear in the dura at the level of the spine ${ }^{18}$. SIH is likely to present with a favorable clinical course and can resolve spontaneously or with a minimally invasive treatment (e.g., simple bed rest and epidural blood patch), but some complications such as chronic subdural hemorrhage $(\mathrm{SDH})^{3,8,10,11,17)}$ and cerebral venous thrombosis, ${ }^{6,17)}$ are reported occasionally.

Chronic SDH is a common intracranial hemorrhage, but is often underestimated compared to other intracranial hemorrhages due to its benign course. Nevertheless, when associated with SIH and improperly treated, it may result in fatal conse- quences such as acute SDH development.

In this report, we present a unique case of chronic SDH associated with SIH which developed into extended pneumocephalus, and we also discuss pathophysiologic considerations.

\section{CASE REPORT}

A 59-year-old male with a 1-month history of progressive postural headache and posterior neck pain visited the emergency room. He had no history of previous illness, minor trauma, or spine procedure. On neurological examination, he was alert and showed no abnormal neurological signs except for slight nuchal rigidity. Initial brain computed tomography (CT) showed lower subdural attenuating crescentic form fluid collection along the bilateral frontotemporoparietal area, which is typical of a SDH (Fig. 1). He was diagnosed with bilateral chronic SDH. In sequence, burr-hole trephination to evacuate the SDH was carried out. Surgical finding was similar to previous chronic SDH one, and after saline irrigation, efforts were made to fill the subdural

- Received : July 6, 2015 • Revised : October 12, 2015 • Accepted : November 11, 2015

- Address for reprints : Seung Hwan Lee, M.D., Ph.D.

Deapartment of Neurosurgery, Kyung Hee University Hospital at Gangdong, College of Medicine, Kyung Hee University, 150 Seongan-ro, Gangdong-gu, Seoul 05355, Korea

Tel : +82-2-440-6286, Fax : +82-2-440-8404, E-mail : toast2000@hanmail.net

- This is an Open Access article distributed under the terms of the Creative Commons Attribution Non-Commercial License (http://creativecommons.org/licenses/by-nc/3.0) which permits unrestricted non-commercial use, distribution, and reproduction in any medium, provided the original work is properly cited. 


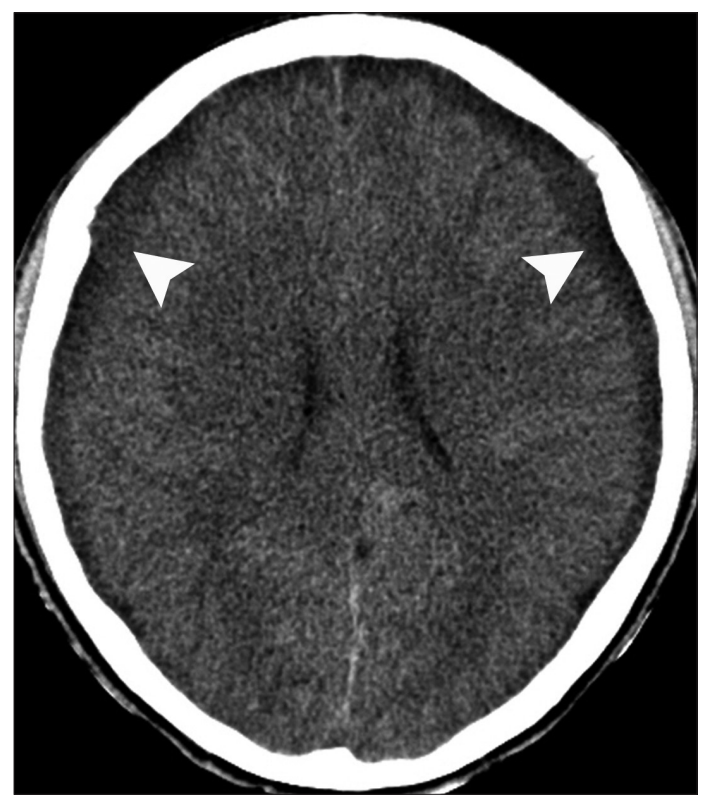

Fig. 1. Initial brain computed tomography obtained at admission showing a chronic subdural hemorrhage (arrowheads) over both cerebral hemispheres, which compressed the adjacent brain. space with saline to reduce pneumocephalus. Lastly, a drainage catheter was placed into the subdural space to drain the remaining SDH and air in the hematoma cavity. The procedure was uneventful. However, postoperative CT taken in 1 hour after surgery revealed an unusually extensive pneumocephalus that occupied both the frontal area, the subarachnoid space of both sylvian, and the basal cistern (Fig. 2). The patient awoke with no neurologic deficit, but he remained lethargic and complained of a severe headache. Brain magnetic resonance imaging (MRI) was performed 3 days later, and showed bilateral re-accumulation of subdural fluid collection and downward displacement of the midline structures with the brainstem, called "sagging" (Fig. 3). We suspected that CSF leakage was the underlying cause of intracranial hypotension and chronic SDH collection; radionuclide cisternography was performed with a lumbar intrathecal injection and whole cerebrospinal axis planar images were obtained $0.5,1,3$, and 5 hours after injection. The 3-hour image revealed CSF leakages at the right distal sacral area (Fig. 4). Subsequently, an epidural blood patch (EBP) was performed via sacral hiatus using a 20 -gauge needle and $10 \mathrm{~mL}$ of autologous blood was injected at the right S1-2 level. Despite strict bed rest
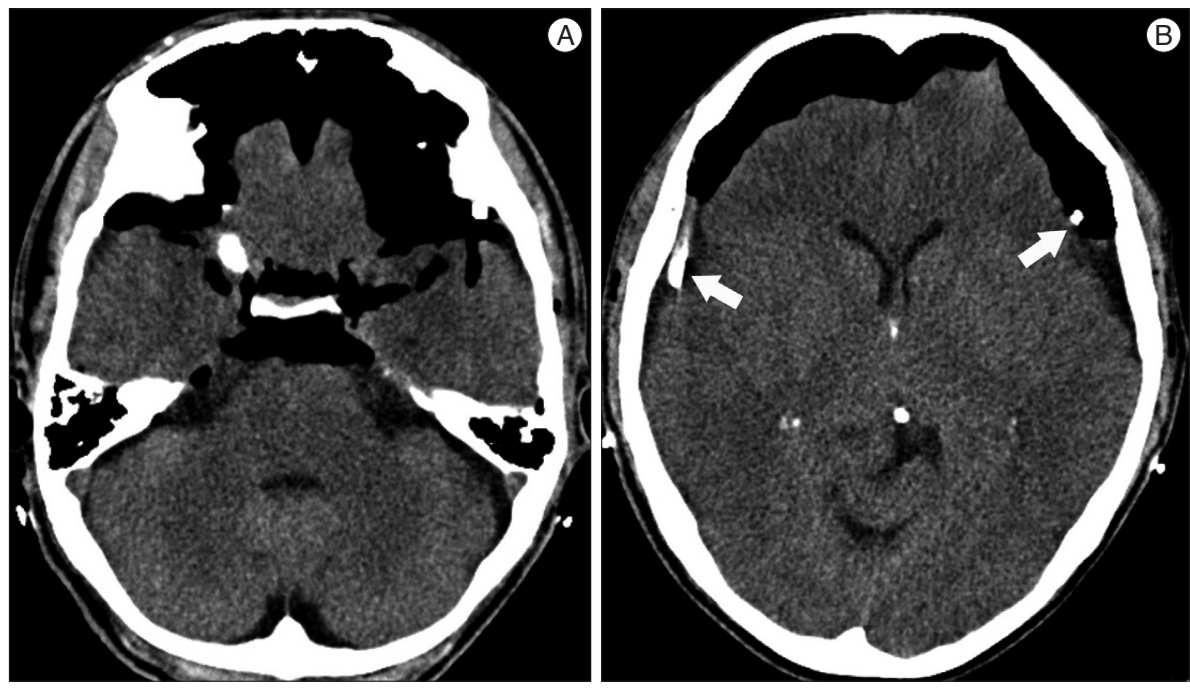

B
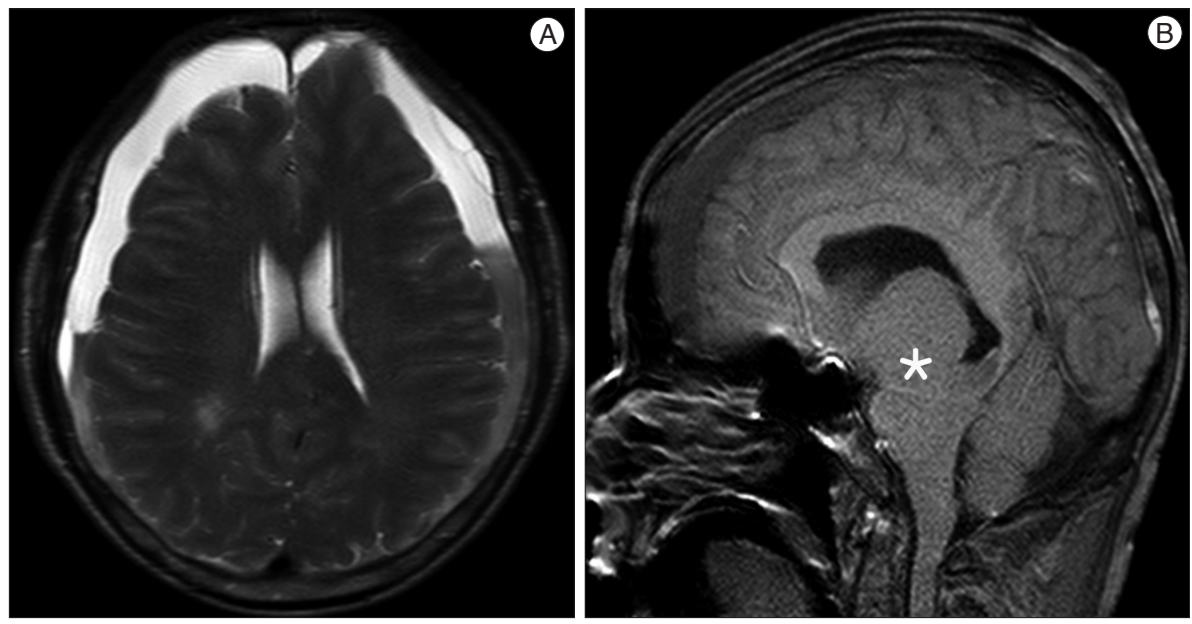

B

Fig. 3. Brain magnetic resonance imaging obtained 3 days after surgical drainage of the subdural hemorrhage. T2-wighted (A) axial image revealing re-accumulation of fluid content mixed with acute and chronic hemorrhage. T1weighted sagittal image (B) showing sagging of the brain stem (asterisk). 
for several days, the patient's headache persisted and did not improve. He became increasingly lethargic a week after the epidural blood patch and a follow-up brain CT scan revealed SDH enlargement with transtentorial herniation of the right-side parahippocampal gyrus (Fig. 5). We assumed that possible dural tear was too large for an epidural blood patch to seal the CSF leakage and that continuous leakage resulted in recurrence of the SDH and transtentorial herniation. To relieve the elevated intracranial pressure from the recurred $\mathrm{SDH}$, we performed burrhole trephination with hematoma evacuation. After surgery, the patient recovered with no neurological deficit, but postoperative brain CT scan showed similar degree of pneumocephalus as previous one, and remained lethargic for a couple of days. Afterwards, lumbar MRI was performed for exact anatomical evaluation of the leaking location, and revealed a large lobulating cystic lesion with a fistula in the sacral canal and right sacral foramen at the S2-upper S3 level, which were compatible with a large meningeal cyst (Fig. 6). A week after the 2nd subdural hematoma surgery, he was brought to the operating room for spine surgery to seal CSF leaking point on the meningeal cyst in S23. After drilling away of the right $\mathrm{S} 2-3$ posterior element, a large meningeal cyst was exposed and found to be connected with the right S3 root. There was a large dural defect on the inferior-lateral aspect of the meningeal cyst. The meningeal cyst was opened

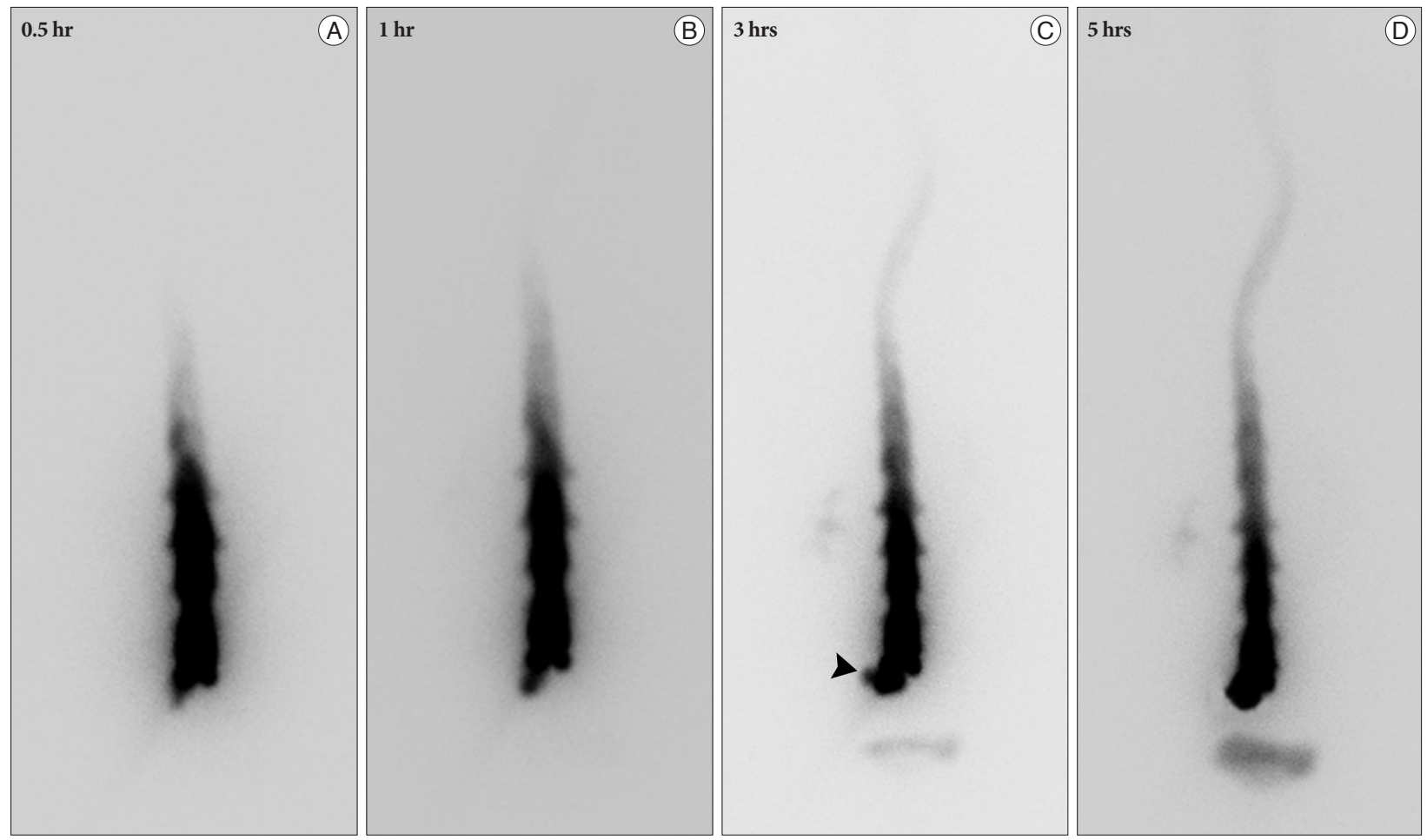

Fig. 4. Radionuclide cisternography showing CSF leakage at the right distal sacral area (arrowhead) on the 3 hours image (A-D).

Fig. 5. Brain CT scans obtained 7 days after epidural blood patch demonstrating effacement of brain stem contour implying transtentorial herniation $(A)$ and increased subdural hemorrhage (B).
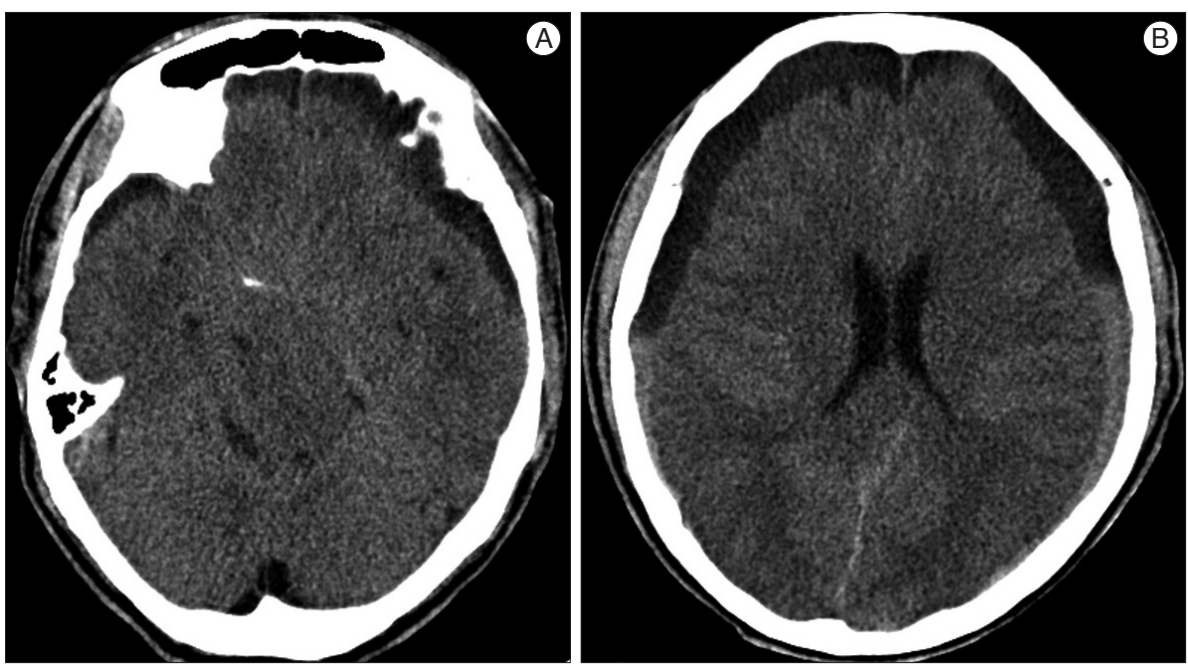
and packed with fascia and gelform, and finally sealed with fibrin glue, instead of ligating the cyst for the preservation of S3 root. The patient recovered without complications, and he was alert and well-oriented the day after surgery. Follow-up brain CT obtained 10 days after spinal surgery indicated that the subdural hematoma had resolved (Fig. 7) and the patient was doing well at the 6-month follow-up examination.

\section{DISCUSSION}

Chronic SDHs are relatively common in the elderly population and $\mathrm{SDH}$ pathophysiology has been considered a consequence of subdural space development due to age and cortical atrophy leading to the formation of a membrane around the hemato$\mathrm{ma}^{12-14)}$. Although there is very little agreement about the optimal treatment strategy to manage chronic subdural hematomas, surgical treatment of symptomatic chronic SDH by a burr-hole procedure or craniotomy, with or without placement of a subdural drain, has been traditionally accepted ${ }^{20)}$.

Usually, the cause of chronic SDH is attributed to minor head
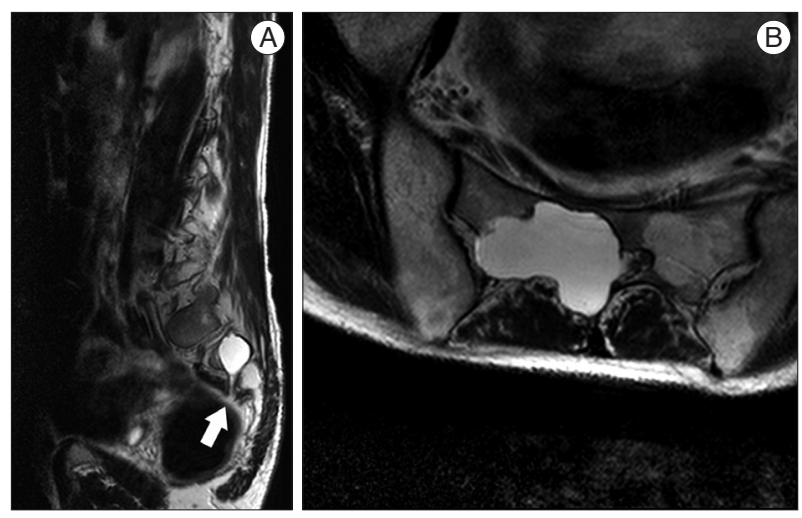

Fig. 6. T2-weighted sagittal (A) and axial (B) magnetic resonance images of the lumbar spine revealing a large lobulating cystic lesion in the sacral canal and right sacral foramen at the S2-upper S3 level with a fistula (arrow), which is supposed to be CSF leaking site. trauma in addition to some risk factors such as coagulopathy and brain atrophy ${ }^{1,15)}$. However, in recent years, SIH is often reported to be associated with chronic SDH development. It is important to differentiate chronic SDH patients with underlying SIH from those without SIH because of the risk of recurrence despite repeated drainage $^{10)}$.

The SIH, first described by Schaltenbrand in $1938^{21)}$, is now believed to be caused by CSF leakage through small dural defects of the spine within the spinal epidural space, resulting in low CSF volume ${ }^{22)}$. SIH is usually characterized by spontaneous postural headaches with neck stiffness, nausea, vomiting, tinnitus, and vertigo and $\mathrm{SIH}$ is defined by a CSF opening pressure lower than $60 \mathrm{~mm} \mathrm{H}_{2} \mathrm{O}^{2,5,16,19)}$. To understand $\mathrm{SDH}$ formation resulting from underlying $\mathrm{SIH}$, Nardone et al. ${ }^{17)}$ gathered previous reports and summarized that downward displacement of the brain due to low CSF pressure may produce tears in bridging veins of the dural border cell layer, causing these veins to rupture. Conversely, as subdural CSF collections gradually enlarge the subdural space, the bridging veins may stretch, and, in some cases, rupture.

The incidence of chronic SDH among SIH patients is reported to range between 20 and $40 \%{ }^{10,11,23)}$. However, there is a lack of established or accepted management guidelines for chronic SDH patients with concomitant SIH. Some reports expressed that measures to stop CSF leakage, specifically epidural blood patches, are required to precede surgical drainage of chronic $\mathrm{SDH}^{3,9)}$. In contrast one report indicated that $\mathrm{SIH}$ resolved spontaneously after surgical drainage of chronic $\mathrm{SDH}^{8)}$.

Surgical drainage of SDH was initially attempted to treat chronic SDH with SIH in our patient to relieve increased intracranial pressure and a drainage catheter was placed to evacuate the remaining SDH and entrapped air. However, unfortunately, air entered the cranial cavity via the drainage catheter which caused profound pneumocephalus. This phenomenon could be explained by the "inverted-soda-bottle effect" proposed by Horowitz $^{7)}$. Horowitz proposed that negative intracranial pressure re-
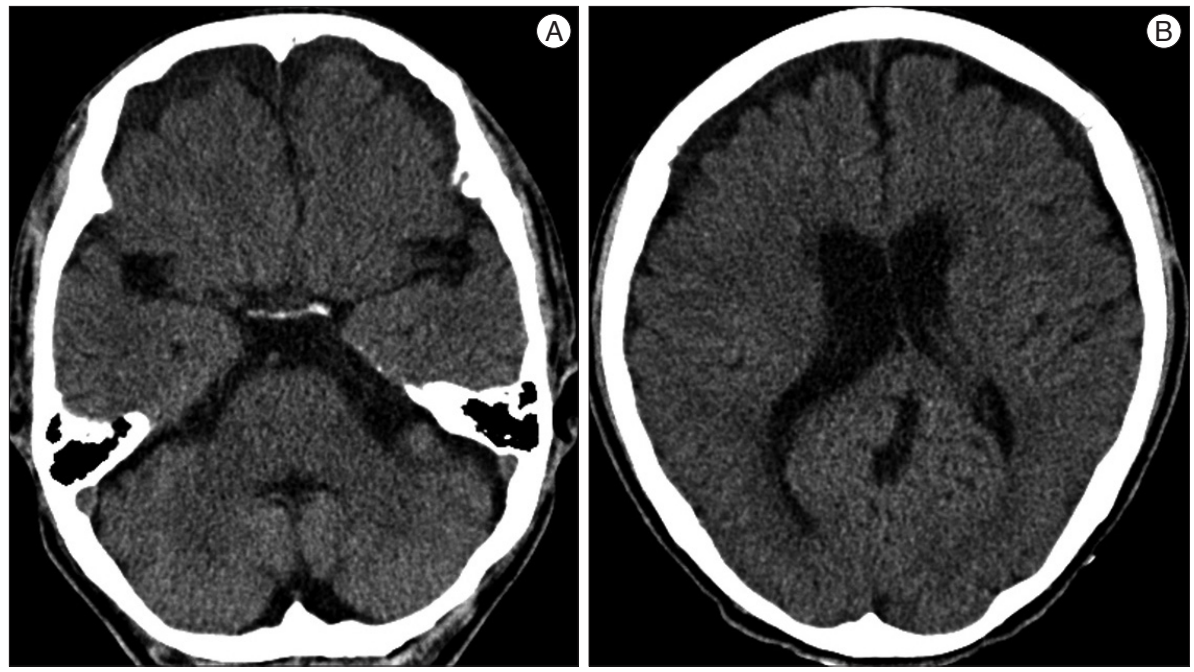

Fig. 7. Follow-up Brain CT scans 10 days after spine surgery showing restoration of brain stem contour $(A)$ and reduced subdural hemorrhage (B). 

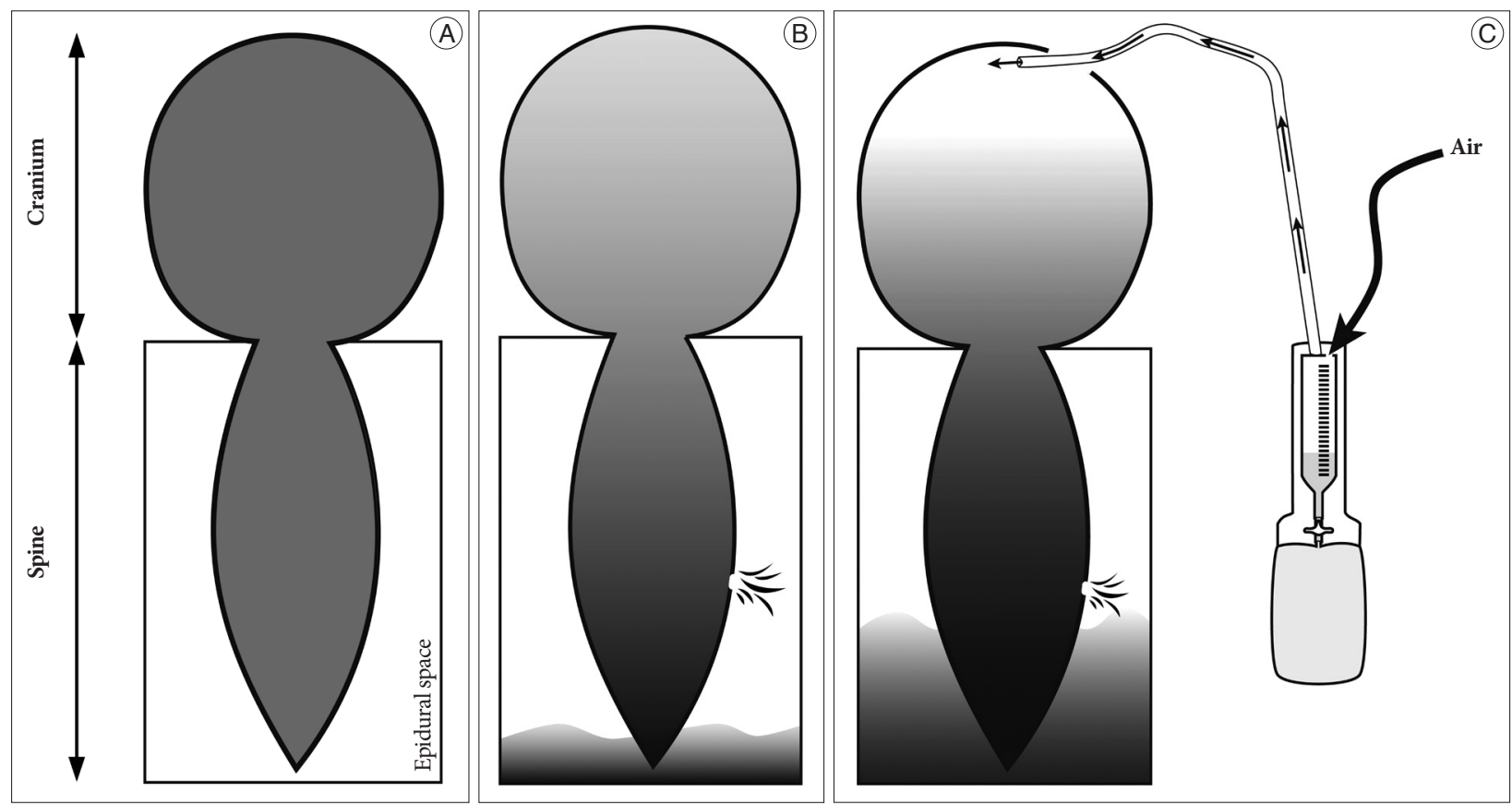

Fig. 8. Schematic drawings showing the mechanism of extensive pneumocephalus development following burrhole drainage when spinal dural defect exist. A : Schematic drawing of the normal status of the body. B : When spinal dural defect exists, cerebrospinal fluid (CSF) leak into the epidural space, leading to lowering intracranial pressure. $C$ : In the presence of drainage catheter connecting between the intracranial and outer space, air within the drainage cylinder may be drawn into the intracranial space; causing CSF to leak continuously, and, extensive pneumocephalus may finally be developed. Note that the drainage cylinder had a hole on top of the cylinder for the air flowing through.

sults from excessive loss of CSF through an iatrogenic lumbar drain or settling into the distendable spinal subarachnoid space or simply drainage via normal pathway physiologic activities, such as inspiration or the Valsalva maneuver. However, when there are fistulous connections between the intracranial and outer spaces, air can enter the intracranial space in response to a negative pressure gradient. With a leakage in the spinal dura, which is an underlying cause of $\mathrm{SIH}$, the fistulous connection was provided by the burr-hole drainage and the additional drainage catheter insertion. Finally, air might enter the intracranial space through the burr hole and the drainage catheter due to negative intracranial pressure, which was previously produced by the CSF leak caused by the spinal dura defect. Schematic drawings of these findings are presented in Fig. 8.

Although an EBP before surgical drainage of SDH could prevent such extensive pneumocephalus, SDH could recur soon after the procedure. Because the dural defect was too large to be sealed or compressed by a mere EBP, SIH was difficult to treat. In addition, there could be a chance to aggravate the CSF leakage in this patient during EBP via sacral hiatus, where the meningeal cyst located in nearby.

Although SIH is not easily determined to be the underlying cause of symptomatic chronic SDH, it should be in the differential diagnosis in identifying the cause of chronic SDH. When profound pneumocephalus is developed after burr-hole trephination for draining chronic SDH and $\mathrm{SIH}$ is suggestive, measures to localize the site of the leak and stop CSF leakage by EBP or repairing the dural defect should be performed. Neverthe- less, in particular cases, performing emergency surgery to drain chronic SDH could be necessary in patients with impending brain herniation. This case report draws attention to the fact that a large spinal dural defect, the cause of SIH, should have been repaired prior to draining chronic $\mathrm{SDH}$

\section{References}

1. Ducruet AF, Grobelny BT, Zacharia BE, Hickman ZL, DeRosa PL, Andersen $\mathrm{KN}$, et al. : The surgical management of chronic subdural hematoma. Neurosurg Rev 35 : 155-169; discussion 169, 2012

2. Fishman RA, Dillon WP : Dural enhancement and cerebral displacement secondary to intracranial hypotension. Neurology 43 (3 Pt 1) : 609611,1993

3. García-Morales I, Porta-Etessam J, Galán L, Lagares A, Molina JA : Recurrent subdural haematomas in a patient with spontaneous intracranial hypotension. Cephalalgia $21:$ 703-705, 2001

4. Gordon N : Spontaneous intracranial hypotension. Dev Med Child Neurol 51 : 932-935, 2009

5. Güler S, Çağlı B, Utku U, Ünlü E, Çelik Y : Intracranial hypotension is a rare cause of orthostatic headache : a review of the etiology, treatment and prognosis of 13 cases. Agri 25 : 69-77, 2013

6. Güler S, Deniz Ç, Utku U, Kehaya S : A case of cerebral venous thrombosis accompanying with intracranial hypotension : headache that changing character. Agri $25: 141-144,2013$

7. Horowitz $\mathrm{M}$ : Intracranial pneumocoele. An unusual complication following mastoid surgery. J Laryngol Otol 78 : 128-134, 1964

8. Ikeda N, Wakabayashi S, Nagao K, Ichioka T, Srivatanakul K, Kajikawa $\mathrm{H}$, et al. : [Two cases of spontaneous intracranial hypotension associated with chronic subdural hematoma only treated with burr hole irrigation of the hematomas]. No To Shinkei 57 : 701-707, 2005

9. Inamasu J, Nakamura Y, Orii M, Saito R, Kuroshima Y, Mayanagi K, et 
al. : Treatment of spontaneous intracranial hypotension secondary to C-2 meningeal cyst by surgical packing--case report. Neurol Med Chir (Tokyo) $44:$ 326-330, 2004

10. Kuramae T, Inamasu J, Nakagawa Y, Nakatsukasa M : Spontaneous intracranial hypotension presenting without orthostatic headache complicated by acute subdural hematoma after drainage for chronic subdural hematoma--case report. Neurol Med Chir (Tokyo) 51 : 518-521, 2011

11. Lai TH, Fuh JL, Lirng JF, Tsai PH, Wang SJ : Subdural haematoma in patients with spontaneous intracranial hypotension. Cephalalgia 27 : 133-138, 2007

12. Lee JY, Ebel H, Ernestus RI, Klug N : Various surgical treatments of chronic subdural hematoma and outcome in 172 patients : is membranectomy necessary? Surg Neurol 61 : 523-527; discussion 527-528, 2004

13. Lee KS : Natural history of chronic subdural haematoma. Brain Inj 18 : $351-358,2004$

14. Lee KS, Bae WK, Doh JW, Bae HG, Yun IG : Origin of chronic subdural haematoma and relation to traumatic subdural lesions. Brain Inj 12 : 901-910, 1998

15. Markwalder TM : Chronic subdural hematomas : a review. J Neurosurg $54: 637-645,1981$

16. Morioka T, Aoki T, Tomoda Y, Takahashi H, Kakeda S, Takeshita I, et al. : Cerebrospinal fluid leakage in intracranial hypotension syndrome : use- fulness of indirect findings in radionuclide cisternography for detection and treatment monitoring. Clin Nucl Med 33 : 181-185, 2008

17. Nardone R, Caleri F, Golaszewski S, Ladurner G, Tezzon F, Bailey A, et al. : Subdural hematoma in a patient with spontaneous intracranial hypotension and cerebral venous thrombosis. Neurol Sci 31 : 669-672, 2010

18. Nosik WA : Intracranial hypotension secondary to lumbar nerve sleeve tear. J Am Med Assoc 157 : 1110-1111, 1955

19. Rando TA, Fishman RA : Spontaneous intracranial hypotension : report of two cases and review of the literature. Neurology 42 (3 Pt 1) : 481-487, 1992

20. Safain M, Roguski M, Antoniou A, Schirmer CM, Malek AM, Riesenburger R: A single center's experience with the bedside subdural evacuating port system : a useful alternative to traditional methods for chronic subdural hematoma evacuation. J Neurosurg 118 : 694-700, 2013

21. Schaltenbrand $G:$ New perspective on the pathophysiology of CSF flow. Zentrabl Neurochir $3: 290-300,1938$

22. Schievink WI : Spontaneous spinal cerebrospinal fluid leaks : a review. Neurosurg Focus 9 : e8, 2000

23. Schievink WI, Maya MM, Moser FG, Tourje J : Spectrum of subdural fluid collections in spontaneous intracranial hypotension. J Neurosurg 103 : 608-613, 2005 